\title{
PROPERTIES OF CERTAIN INTEGRAL OPERATORS
}

\author{
SHIGEYOSHI OWA
}

\begin{abstract}
Two integral operators $P^{\alpha}$ and $Q_{\beta}^{\alpha}$ for analytic functions in the open unit disk are introduced. The object of the present paper is to derive some properties of integral operators $P^{\alpha}$ and $Q_{\beta}^{\alpha}$.
\end{abstract}

\section{INTRODUCTION}

Let $A$ be the class of functions of the form

$$
f(z)=z+\sum_{n=2}^{\infty} a_{n} z^{n}
$$

which are analytic in the open unit disk $U=\{z:|z|<1\}$. Recently, Jung, Kim, and Srivastava [1] have introduced the following one-parameter families of integral operators:

$$
\begin{gathered}
P^{\alpha} f=P^{\alpha} f(z)=\frac{2^{\alpha}}{z \Gamma(\alpha)} \int_{0}^{z}\left(\log \frac{z}{t}\right)^{\alpha-1} f(t) d t \quad(\alpha>0), \\
Q_{\beta}^{\alpha} f=Q_{\beta}^{\alpha} f(z)=\left(\frac{\alpha+\beta}{\beta}\right) \frac{\alpha}{z^{\beta}} \int_{0}^{z}\left(1-\frac{t}{z}\right)^{\alpha-1} t^{\beta-1} f(t) d t \\
(\alpha>0, \beta>-1)
\end{gathered}
$$

and

$$
J_{\alpha} f=J_{\alpha} f(z)=\frac{\alpha+1}{z^{\alpha}} \int_{0}^{z} t^{\alpha-1} f(t) d t \quad(\alpha>-1),
$$

1991 Mathematics Subject Classification. 30C45.

Key words and phrases. Analytic functions, logarithmic differentiation, integral operator. 
where $\Gamma(\alpha)$ is the familiar Gamma function, and (in general)

$$
\left[\begin{array}{l}
\alpha \\
\beta
\end{array}\right]=\frac{\Gamma(\alpha+1)}{\Gamma(\alpha-\beta+1) \Gamma(\beta+1)}=\left[\begin{array}{c}
\alpha \\
\alpha-\beta
\end{array}\right] .
$$

For $\alpha \in \mathbb{N}=\{1,2,3, \ldots\}$, the operators $P^{\alpha}, Q_{1}^{\alpha}$, and $J_{\alpha}$ were considered by Bernardi ([2], [3]). Further, for a real number $\alpha>-1$, the operator $J_{\alpha}$ was used by Owa and Srivastava [4], and by Srivastava and Owa ([8], [6]).

Remark 1. For $f(z) \in A$ given by (1.1), Jung, Kim, and Srivastava [1] have shown that

$$
\begin{gathered}
P^{\alpha} f(z)=z+\sum_{n=2}^{\infty}\left(\frac{2}{n+1}\right)^{\alpha} a_{n} z^{n} \quad(\alpha>0), \\
Q_{\beta}^{\alpha} f(z)=z+\frac{\Gamma(\alpha+\beta+1)}{\Gamma(\beta+1)} \sum_{n=2}^{\infty} \frac{\Gamma(\beta+n)}{\Gamma(\alpha+\beta+n)} a_{n} z^{n} \\
(\alpha>0, \beta>-1)
\end{gathered}
$$

and

$$
J_{\alpha} f(z)=z+\sum_{n=2}^{\infty}\left(\frac{\alpha+1}{\alpha+n}\right) a_{n} z^{n} \quad(\alpha>-1) .
$$

By virtue of (1.6) and (1.8), we see that

$$
J_{\alpha} f(z)=Q_{\alpha}^{1} f(z) \quad(\alpha>-1) .
$$

\section{An Applichtion of the Miller-Mocanu Lemma}

To derive some properties of operators, we have to recall here the following lemma due to Miller and Mocanu [7].

Lemma 1. Let $w(u, v)$ be a complex valued function,

$$
w: D \rightarrow \mathbb{C}, D \subset \mathbb{C} \times \mathbb{C}(\mathbb{C} \text { is the complex plane }),
$$

and let $u=u_{1}+i u_{2}$ and $v=v_{1}+i v_{2}$. Suppose that the function $w(u, v)$ satisfies the following conditions:

(i) $w(u, v)$ is continuous in $D$;

(ii) $(1,0) \in D$ and $\operatorname{Re}\{w(1,0)\}>0$;

(iii) $\operatorname{Re}\left\{w\left(i u_{2}, v_{1}\right)\right\} \leq 0$ for all $\left(i u_{2}, v_{1}\right) \in D$ and such that $v_{1} \leq-(1+$ $\left.u_{2}^{2}\right) / 2$.

Let $p(z)$ be regular in $U$ and $p(z)=1+p_{1} z+p_{2} z^{2}+\ldots$ such that $\left(p(z), z p^{\prime}(z)\right) \in D$ for all $z \in U$. If $\operatorname{Re}\left\{w\left(p(z), z p^{\prime}(z)\right)\right\}>0(z \in U)$, then $\operatorname{Re}\{p(z)\}>0(z \in U)$.

Applying the above lemma, we derive 
Theorem 1. If $f(z) \in A$ satisfies

$$
\operatorname{Re}\left\{\frac{P^{\alpha-2} f(z)}{P^{\alpha-1} f(z)}\right\}>\beta \quad(\alpha>2 ; z \in U)
$$

for some $\beta(\beta<1)$, then

$$
\operatorname{Re}\left\{\frac{P^{\alpha-1} f(z)}{P^{\alpha} f(z)}\right\}>\frac{4 \beta-1+\sqrt{16 \beta^{2}-8 \beta+17}}{8}(z \in U) .
$$

Proof. Noting that

$$
z\left(P^{\alpha} f(z)\right)^{\prime}=2 P^{\alpha-1} F(z)-P^{\alpha} f(z) \quad(\alpha>1),
$$

we have

$$
\frac{z\left(P^{\alpha} f(z)\right)^{\prime}}{P^{\alpha} f(z)}=2 \frac{P^{\alpha-1} f(z)}{P^{\alpha} f(z)}-1 \quad(\alpha>1) .
$$

Define the function $p(z)$ by

$$
\frac{P^{\alpha-1} f(z)}{P^{\alpha} f(z)}=\gamma+(1-\gamma) p(z)
$$

with

$$
\gamma=\frac{4 \beta-1+\sqrt{16 \beta^{2}-8 \beta+17}}{8} .
$$

Then $p(z)=1+p_{1} z+p_{2} z^{2}+\ldots$ is analytic in the open unit disk $U$. Since

$$
\frac{z\left(P^{\alpha-1} f(z)\right)^{\prime}}{P^{\alpha-1} f(z)}-\frac{z\left(P^{\alpha} f(z)\right)^{\prime}}{P^{\alpha} f(z)}=\frac{(1-\gamma) z p^{\prime}(z)}{\gamma+(1-\gamma) p(z)},
$$

or

$$
\frac{P^{\alpha-2} f(z)}{P^{\alpha-1} f(z)}=\frac{P^{\alpha-1} f(z)}{P^{\alpha} f(z)}+\frac{(1-\gamma) z p^{\prime}(z)}{2\{\gamma+(1-\gamma) p(z)\}}
$$

we have

$$
\begin{gathered}
\operatorname{Re}\left\{\frac{P^{\alpha-2} f(z)}{P^{\alpha-1} f(z)}-\beta\right\}= \\
=\operatorname{Re}\left\{\gamma+(1-\gamma) p(z)+\frac{(1-\gamma) z p^{\prime}(z)}{2\{\gamma+(1-\gamma) p(z)\}}-\beta\right\}>0 .
\end{gathered}
$$

Therefore, if we define the function $w(u, v)$ by

$$
w(u, v)=\gamma-\beta+(1-\gamma) u+\frac{(1-\gamma) v}{2\{\gamma+(1-\gamma) u\}},
$$

then we see that 
(i) $w(u, v)$ is continuous in $D=\left(C-\left\{\frac{\gamma}{\gamma-1}\right\}\right) \times C$;

(ii) $(1,0) \in D$ and $\operatorname{Re}\{w(1,0)\}=1-\beta>0$;

(iii) for all $\left(i u_{2}, v_{1}\right) \in D$ and such that $v_{1} \leq-\left(1+u_{2}^{2}\right) / 2$,

$$
\begin{aligned}
\operatorname{Re}\left\{w\left(i u_{2}, v_{1}\right)\right\} & =\gamma-\beta+\frac{\gamma(1-\gamma) v_{1}}{2\left\{\gamma^{2}+(1-\gamma)^{2} u_{2}^{2}\right\}} \leq \\
& \leq \gamma-\beta-\frac{\gamma(1-\gamma)\left(1+u_{2}^{2}\right)}{4\left\{\gamma^{2}+(1-\gamma)^{2} u_{2}^{2}\right\}}= \\
& =-\frac{(1-\gamma)(\gamma+4(1-\gamma) \beta)}{4\left\{\gamma^{2}+(1-\gamma)^{2} u_{2}^{2}\right\}} u_{2}^{2} \leq 0 .
\end{aligned}
$$

This implies that the function $w(u, v)$ satisfies the conditions in Lemma 1. Thus, applying Lemma 1, we conclude that

$$
\begin{gathered}
\operatorname{Re}\left\{\frac{P^{\alpha-1} f(z)}{P^{\alpha} f(z)}\right\}>\gamma= \\
=\frac{4 \beta-1+\sqrt{16 \beta^{2}-8 \beta+17}}{8}(z \in U) .
\end{gathered}
$$

Taking the special values for $\beta$ in Theorem 1 , we have

Corollary 1. Let $f(z)$ be in the class A. Then

(i) $\operatorname{Re}\left\{\frac{P^{\alpha-2} f(z)}{P^{\alpha-1} f(z)}\right\}>-\frac{1}{2} \quad(z \in U)$

$$
\Longrightarrow \operatorname{Re}\left\{\frac{P^{\alpha-1} f(z)}{P^{\alpha} f(z)}\right\}>\frac{1}{4} \quad(z \in U),
$$

(ii) $\operatorname{Re}\left\{\frac{P^{\alpha-2} f(z)}{P^{\alpha-1} f(z)}\right\}>-\frac{1}{4} \quad(z \in U)$

$$
\Longrightarrow \operatorname{Re}\left\{\frac{P^{\alpha-1} f(z)}{P^{\alpha} f(z)}\right\}>\frac{\sqrt{5}-1}{4} \quad(z \in U),
$$

(iii) $\operatorname{Re}\left\{\frac{P^{\alpha-2} f(z)}{P^{\alpha-1} f(z)}\right\}>0 \quad(z \in U)$

$$
\Longrightarrow \operatorname{Re}\left\{\frac{P^{\alpha-1} f(z)}{P^{\alpha} f(z)}\right\}>\frac{\sqrt{17}-1}{8} \quad(z \in U),
$$

(iv) $\operatorname{Re}\left\{\frac{P^{\alpha-2} f(z)}{P^{\alpha-1} f(z)}\right\}>\frac{1}{4} \quad(z \in U)$

$$
\Longrightarrow \operatorname{Re}\left\{\frac{P^{\alpha-1} f(z)}{P^{\alpha} f(z)}\right\}>\frac{1}{2} \quad(z \in U),
$$

and

(v) $\operatorname{Re}\left\{\frac{P^{\alpha-2} f(z)}{P^{\alpha-1} f(z)}\right\}>\frac{1}{2} \quad(z \in U)$

$$
\Longrightarrow \operatorname{Re}\left\{\frac{P^{\alpha-1} f(z)}{P^{\alpha} f(z)}\right\}>\frac{\sqrt{17}+1}{8} \quad(z \in U) .
$$


Next, we have

Theorem 2. If $f(z) \in A$ satisfies

$$
\operatorname{Re}\left\{\frac{Q_{\beta}^{\alpha-2} f(z)}{Q_{\beta}^{\alpha-1} f(z)}\right\}>\gamma(\alpha>2, \beta>-1 ; z \in U)
$$

for some $\gamma((\alpha+\beta-3) / 2(\alpha+\beta-1) \leq \gamma<1)$, then

$$
\operatorname{Re}\left\{\frac{Q_{\beta}^{\alpha-1} f(z)}{Q_{\beta}^{\alpha} f(z)}\right\}>\delta(z \in U)
$$

where

$$
\delta=\frac{1+2 \gamma(\alpha+\beta-1)+\sqrt{(1+2 \gamma(\alpha+\beta-1))^{2}+8(\alpha+\beta)}}{4(\alpha+\beta)} .
$$

Proof. By the definition of $Q_{\beta}^{\alpha} f(z)$, we know that

$$
z\left(Q_{\beta}^{\alpha} f(z)\right)^{\prime}=(\alpha+\beta) Q_{\beta}^{\alpha-1} f(z)-(\alpha+\beta-1) Q_{\beta}^{\alpha} f(z)
$$

$$
(\alpha>1, \beta>-1)
$$

so that

$$
\frac{z\left(Q_{\beta}^{\alpha} f(z)\right)^{\prime}}{Q_{\beta}^{\alpha} f(z)}=(\alpha+\beta) \frac{Q_{\beta}^{\alpha-1} f(z)}{Q_{\beta}^{\alpha} f(z)}-(\alpha+\beta-1) .
$$

We define the function $p(z)$ by

$$
\frac{Q_{\beta}^{\alpha-1} f(z)}{Q_{\beta}^{\alpha} f(z)}=\delta+(1-\delta) p(z)
$$

Then $p(z)=1+p_{1} z+p_{2} z^{2}+\ldots$ is analytic in $U$. Making use of the logarithmic differentiations in both sides of (2.17), we have

$$
\frac{z\left(Q_{\beta}^{\alpha-1} f(z)\right)^{\prime}}{Q_{\beta}^{\alpha-1} f(z)}=\frac{z\left(Q_{\beta}^{\alpha} f(z)\right)^{\prime}}{Q_{\beta}^{\alpha} f(z)}+\frac{(1-\delta) z p^{\prime}(z)}{\delta+(1-\delta) p(z)} .
$$

Applying (2.15) to (2.18), we obtain that

$$
\begin{aligned}
& \frac{Q_{\beta}^{\alpha-2} f(z)}{Q_{\beta}^{\alpha-1} f(z)}=\frac{1}{\alpha+\beta-1}\left\{(\alpha+\beta) \frac{Q_{\beta}^{\alpha-1} f(z)}{Q_{\beta}^{\alpha} f(z)}-1+\right. \\
& \left.+\frac{(1-\delta) z p^{\prime}(z)}{\delta+(1-\delta) p(z)}\right\}=\frac{1}{\alpha+\beta-1}\{\delta(\alpha+\beta)-1+ \\
& \left.\quad+(\alpha+\beta)(1-\delta) p(z)+\frac{(1-\delta) z p^{\prime}(z)}{\delta+(1-\delta) p(z)}\right\},
\end{aligned}
$$


that is, that

$$
\begin{gathered}
\operatorname{Re}\left\{\frac{Q_{\beta}^{\alpha-2} f(z)}{Q_{\beta}^{\alpha-1} f(z)}-\gamma\right\}=\frac{1}{\alpha+\beta-1} \operatorname{Re}\{\delta(\alpha+\beta)-1+ \\
\left.+(\alpha+\beta)(1-\delta) p(z)+\frac{(1-\delta) z p^{\prime}(z)}{\delta+(1-\delta) p(z)}\right\}-\gamma>0 .
\end{gathered}
$$

Now, we let

$$
\begin{aligned}
& w(u, v)=\frac{1}{\alpha+\beta-1}\{\delta(\alpha+\beta)-1+ \\
& \left.+(\alpha+\beta)(1-\delta) u+\frac{(1-\delta) v}{\delta+(1-\delta) u}\right\}-\gamma
\end{aligned}
$$

with $u=u_{1}+i u_{2}$ and $v=v_{1}+i v_{2}$. Then $w(u, v)$ satisfies that

(i) $w(u, v)$ is continuous in $D=\left(\mathbb{C}-\left\{\frac{\delta}{\delta-1}\right\}\right) \times \mathbb{C}$;

(ii) $(1,0) \in D$ and $\operatorname{Re}\{w(1,0)\}=1-\gamma>0$;

(iii) for all $\left(i u_{2}, v_{1}\right) \in D$ and such that $v_{1} \leq-\left(1+u_{2}^{2}\right) / 2$,

$$
\begin{gathered}
\operatorname{Re}\left\{w\left(i u_{2}, v_{1}\right)\right\}=\frac{1}{\alpha+\beta-1}\{\delta(\alpha+\beta)-1+ \\
\left.+\frac{\delta(1-\delta) v_{1}}{\delta^{2}+(1-\delta)^{2} u_{2}^{2}}\right\}-\gamma \leq \frac{1}{\alpha+\beta-1}\{\delta(\alpha+\beta)-1- \\
\left.-\gamma(\alpha+\beta-1)-\frac{\delta(1-\delta)\left(1+u_{2}^{2}\right)}{2\left\{\delta^{2}+(1-\delta)^{2} u_{2}^{2}\right\}}\right\} \leq 0 .
\end{gathered}
$$

Thus, the function $w(u, v)$ satisfies the conditions in Lemma 1. This shows that $\operatorname{Re}\{p(z)\}>0$, or

$$
\operatorname{Re}\left\{\frac{Q_{\beta}^{\alpha-1} f(z)}{Q_{\beta}^{\alpha} f(z)}\right\}>\delta \quad(z \in U)
$$

If we take $\gamma=(\alpha+\beta-3) / 2(\alpha+\beta-1)$ in Theorem 2 , then we have

Corollary 2. If $f(z) \in A$ satisfies

$$
\begin{gathered}
\operatorname{Re}\left\{\frac{Q_{\beta}^{\alpha-2} f(z)}{Q_{\beta}^{\alpha-1} f(z)}\right\}>\frac{\alpha+\beta-3}{2(\alpha+\beta-1)} \\
(\alpha>2, \beta>-1 ; z \in U),
\end{gathered}
$$

then

$$
\operatorname{Re}\left\{\frac{Q_{\beta}^{\alpha-1} f(z)}{Q_{\beta}^{\alpha} f(z)}\right\}>\frac{1}{2} \quad(z \in U) .
$$

Further, letting $\alpha=2-\beta$ and $\gamma=1 / 2$ in Theorem 2, we have 
Corollary 3. If $f(z) \in A$ satisfies

$$
\operatorname{Re}\left\{\frac{Q_{\beta}^{-\beta} f(z)}{Q_{\beta}^{1-\beta} f(z)}\right\}>\frac{1}{2} \quad(\beta>-1 ; z \in U),
$$

then

$$
\operatorname{Re}\left\{\frac{Q_{\beta}^{1-\beta} f(z)}{Q_{\beta}^{2-\beta} f(z)}\right\}>\frac{1+\sqrt{5}}{4} \quad(z \in U) .
$$

\section{An Application of JaCK's Lemma}

We need the following lemma due to Jack [8], (also, due to Miller and Mocanu [7]).

Lemma 2. Let $w(z)$ be analytic in $U$ with $w(0)=0$. If $|w(z)|$ attains its maximum value on the circle $|z|=r<1$ at a point $z_{0} \in U$, then we can write

$$
z_{0} w^{\prime}\left(z_{0}\right)=k w\left(z_{0}\right)
$$

where $k$ is a real number and $k \geq 1$.

Applying Lemma 2 for the operator $P^{\alpha}$, we have

Theorem 3. If $f(z) \in A$ satisfies

$$
\operatorname{Re}\left\{\frac{P^{\alpha-2} f(z)}{P^{\alpha-1} f(z)}\right\}>\beta(\alpha>2, \beta \leq 1 / 4 ; z \in U),
$$

then

$$
\operatorname{Re}\left\{\frac{P^{\alpha-1} f(z)}{P^{\alpha} f(z)}\right\}>\gamma \quad(z \in U)
$$

where

$$
\gamma=\frac{3+4 \beta+\sqrt{16 \beta^{2}-40 \beta+9}}{8} .
$$

Proof. Defining the function $w(u, v)$ by

$$
\frac{P^{\alpha-1} f(z)}{P^{\alpha} f(z)}=\frac{1-(1-2 \gamma) w(z)}{1+w(z)}
$$

we see that $w(z)$ is analytic in $U$ and $w(0)=0$. It follows from (3.5) that

$$
\begin{aligned}
& \frac{z\left(P^{\alpha-1} f(z)\right)^{\prime}}{P^{\alpha-1} f(z)}=\frac{z\left(P^{\alpha} f(z)\right)^{\prime}}{P^{\alpha} f(z)}- \\
& -\frac{(1-2 \gamma) z w^{\prime}(z)}{1-(1-2 \gamma) w(z)}-\frac{z w^{\prime}(z)}{1+w(z)} .
\end{aligned}
$$


Using (2.3), we obtain that

$$
\begin{gathered}
\frac{P^{\alpha-2} f(z)}{P^{\alpha-1} f(z)}=\frac{1-(1-2 \gamma) w(z)}{1+w(z)}- \\
-\frac{1}{2} \frac{z w^{\prime}(z)}{w(z)}\left(\frac{(1-2 \gamma) w(z)}{1-(1-2 \gamma) w(z)}+\frac{w(z)}{1+w(z)}\right) .
\end{gathered}
$$

If we suppose that there exists a point $z_{0} \in U$ such that

$$
\max _{|z| \leq\left|z_{0}\right|}|w(z)|=\left|w\left(z_{0}\right)\right|=1 \quad\left(w\left(z_{0}\right) \neq-1\right),
$$

then Lemma 2 gives us that

$$
z_{0} w^{\prime}\left(z_{0}\right)=k w\left(z_{0}\right) \quad(k \geq 1) .
$$

Therefore, letting $w\left(z_{0}\right)=e^{i \theta}(\theta \neq \pi)$, we have

$$
\begin{gathered}
\operatorname{Re}\left\{\frac{P^{\alpha-2} f\left(z_{0}\right)}{P^{\alpha-1} f\left(z_{0}\right)}\right\}=\operatorname{Re}\left\{\frac{1-(1-2 \gamma) w\left(z_{0}\right)}{1+w\left(z_{0}\right)}-\right. \\
\left.-\frac{k}{2}\left(\frac{(1-2 \gamma) w\left(z_{0}\right)}{1-(1-2 \gamma) w\left(z_{0}\right)}+\frac{w\left(z_{0}\right)}{1+w\left(z_{0}\right)}\right)\right\}= \\
=\operatorname{Re}\left\{\frac{1-(1-2 \gamma) e^{i \theta}}{1+e^{i \theta}}-\frac{k}{2}\left(\frac{(1-2 \gamma) e^{i \theta}}{1-(1-2 \gamma) e^{i \theta}}+\frac{e^{i \theta}}{1+e^{i \theta}}\right)\right\}= \\
=\gamma-\frac{k}{2}\left(\frac{(1-2 \gamma)(\cos \theta-(1-2 \gamma))}{1+(1-2 \gamma)^{2}-2(1-2 \gamma) \cos \theta}+\frac{1}{2}\right) \leq \\
\leq \gamma-\frac{k}{2}\left(\frac{1}{2}-\frac{1-2 \gamma}{2(1-\gamma)}\right) \leq \frac{\gamma(3-4 \gamma)}{4(1-\gamma)}=\beta .
\end{gathered}
$$

This contradicts our condition (3.2). Therefore, we have

$$
|w(z)|=\left|\frac{\frac{P^{\alpha-1} f(z)}{P^{\alpha} f(z)}-1}{\frac{P^{\alpha-1} f(z)}{P^{\alpha} f(z)}+(1-2 \gamma)}\right|<1 \quad(z \in U),
$$

which implies (3.3).

Taking $\beta=0$ and $\beta=1 / 4$ in Theorem 3 , we have

Corollary 4. Let $f(z)$ be in the class A. Then

$$
\begin{aligned}
\operatorname{Re}\left\{\frac{P^{\alpha-2} f(z)}{P^{\alpha-1} f(z)}\right\} & >0 \quad(\alpha>2 ; z \in U) \\
& \Longrightarrow \operatorname{Re}\left\{\frac{P^{\alpha-1} f(z)}{P^{\alpha} f(z)}\right\}>\frac{3}{4} \quad(z \in U)
\end{aligned}
$$


and

$$
\begin{aligned}
\operatorname{Re}\left\{\frac{P^{\alpha-2} f(z)}{P^{\alpha-1} f(z)}\right\} & >-\frac{1}{4} \quad(\alpha>2 ; z \in U) \\
& \Longrightarrow \operatorname{Re}\left\{\frac{P^{\alpha-1} f(z)}{P^{\alpha} f(z)}\right\}>\frac{1}{2} \quad(z \in U) .
\end{aligned}
$$

Finally, we prove

Theorem 4. If $f(z) \in A$ satisfies

$$
\operatorname{Re}\left\{\frac{Q^{\alpha-2} f(z)}{Q^{\alpha-1} f(z)}\right\}>\gamma \quad(\alpha>2 ; \beta>-1 ; z \in U)
$$

for some $\gamma(\gamma<1)$, then

$$
\operatorname{Re}\left\{\frac{Q_{\beta}^{\alpha-1} f(z)}{Q_{\beta}^{\alpha} f(z)}\right\}>\delta \quad(z \in U),
$$

where $\delta(0 \leq \delta<1)$ is the smallest positive root of the equation

$$
\begin{gathered}
2(\alpha+\beta) \delta^{2}-\{2(\alpha+\beta)(\gamma+1)- \\
-(2 \gamma-1)\} \delta+2\{(\alpha+\beta-1) \gamma+1\}=0 .
\end{gathered}
$$

Proof. Defining the function $w(z)$ by

$$
\frac{Q_{\beta}^{\alpha-1} f(z)}{Q_{\beta}^{\alpha} f(z)}=\frac{1-(1-2 \delta) w(z)}{1+w(z)},
$$

we obtain that

$$
\begin{gathered}
\frac{Q_{\beta}^{\alpha-2} f(z)}{Q_{\beta}^{\alpha-1} f(z)}=\frac{1}{\alpha+\beta-1}\left\{(\alpha+\beta) \frac{1-(1-2 \delta) w(z)}{1+w(z)}-1-\right. \\
\left.-\frac{z w^{\prime}(z)}{w(z)}\left(\frac{(1-2 \delta) w(z)}{1-(1-2 \delta) w(z)}+\frac{w(z)}{1+w(z)}\right)\right\}
\end{gathered}
$$

Therefore, supposing that there exists a point $z_{0} \in U$ such that

$$
\max _{|z| \leq\left|z_{0}\right|}|w(z)|=\left|w\left(z_{0}\right)\right|=1 \quad\left(w\left(z_{0}\right) \neq-1\right)
$$

we see that

$$
\begin{gathered}
\operatorname{Re}\left\{\frac{Q_{\beta}^{\alpha-2} f\left(z_{0}\right)}{Q_{\beta}^{\alpha-1} f\left(z_{0}\right)}\right\} \leq \\
\leq \frac{1}{\alpha+\beta-1}\left\{(\alpha+\beta) \delta-1-\frac{\delta}{2(1-\delta)}\right\}=\gamma .
\end{gathered}
$$

Making $\gamma=0$ in Theorem 4, we have 
Corollary 5. If $f(z) \in A$ satisfies

$$
\operatorname{Re}\left\{\frac{Q_{\beta}^{\alpha-2} f(z)}{Q_{\beta}^{\alpha-1} f(z)}\right\}>0(\alpha>2 ; \beta>-1 ; z \in U),
$$

then

$$
\operatorname{Re}\left\{\frac{Q_{\beta}^{\alpha-1} f(z)}{Q_{\beta}^{\alpha} f(z)}\right\}>\frac{-1}{\alpha+\beta-1}(z \in U) .
$$

Letting $\gamma=1 / 2$ in Theorem 4 , we have

Corollary 6. If $f(z) \in A$ satisfies

$$
\operatorname{Re}\left\{\frac{Q_{\beta}^{\alpha-2} f(z)}{Q_{\beta}^{\alpha-1} f(z)}\right\}>\frac{1}{2}(\alpha>2 ; \beta>-1 ; z \in U),
$$

then

$$
\operatorname{Re}\left\{\frac{Q_{\beta}^{\alpha-1} f(z)}{Q_{\beta}^{\alpha} f(z)}\right\}>\frac{\alpha+\beta-3}{\alpha+\beta-1}(z \in U) .
$$

Further, if $\alpha=3-\beta$, we have

$$
\begin{aligned}
\operatorname{Re}\left\{\frac{Q_{\beta}^{1-\beta} f(z)}{Q_{\beta}^{2-\beta} f(z)}\right\} & >\frac{1}{2} \quad(\beta>-1 ; z \in U) \\
& \Longrightarrow \operatorname{Re}\left\{\frac{Q_{\beta}^{2-\beta} f(z)}{Q_{\beta}^{3-\beta} f(z)}\right\}>0 \quad(z \in U) .
\end{aligned}
$$

\section{ACKNOWLEDGEMENT}

This research was supported in part by the Japanese Ministry of Education, Science and Culture under a Grant-in-Aid for General Scientific Research (No. 04640204).

\section{REFERENCES}

1. S. D. Bernardi, Convex and starlike univalent functions. Trans. Amer. Math. Soc. 135 (1969), 429-446.

2. S. D. Bernardi, The radius of univalence of certain analytic functions. Proc. Amer. Math. Soc. 24 (1970), 312-318.

3. I. S. Jack, Functions starlike and convex of order $\alpha$, J. London Math. Soc. 3 (1971), 469-474. 
4. I. B. Jung, Y. C. Kim, and H. M. Srivastava, The Hardy space of analytic functions associated with certain one-parameter families of integral operators, to appear.

5. S. S. Miller and P. T. Mocanu, Second-order differential inequalities in the complex plane. J. Math. Anal. Appl. 65 (1978), 289-305.

6. S. Owa and H. M. Srivastava, Some applications of the generalized Libera operator. Proc. Japan Acad. 62 (1986), 125-128.

7. H. M. Srivastava and S. Owa, New characterization of certain starlike and convex generalized hypergeometric functions. J. Natl. Acad. Math. India 3 (1985), 198-202.

8. H. M. Srivastava and S. Owa, A certain one-parameter additive family of operators defined on analytic functions. J. Anal. Appl. 118 (1986), 8087.

(Received 3.06.94)

Author's address:

Department of Mathematics

Kinki University

Higashi-Osaka, Osaka 577

Japan 